\title{
Modeling the Effect of Orientation on Human-Powered Inertial Energy Harvesters
}

\author{
Geoff V. Merrett, Hui Huang, and Neil M. White
}

\begin{abstract}
A fundamental challenge in realizing body-worn sensors is in providing an effective and long-lasting power supply. Issues regarding batteries have prompted researchers to investigate powering devices by extracting energy from the inertial movement of the human body. While previous studies have investigated the effect of generator location and wearer activity on harvestable power, they have not considered the orientation of the generator; this is the focus of this paper. Acceleration data collected across a sample population (ten participants) during different activities (walking and running) and generator location (five locations on the body) are presented. These data are processed to analyze the effect of orientation, and we find that it can significantly reduce the harvestable power. Subsequently, we propose and analyze how two degree-offreedom generators can improve tolerance to rotation; results indicate that it can be improved by one order of magnitude.
\end{abstract}

Index Terms - energy harvesting, body sensor networks, micropower generator

\section{INTRODUCTION}

$\mathrm{D}$ EVELOPMENTS in ubiquitous computing and assistive technologies are expanding the possibilities of body-worn sensing, and commercial interest in such technologies is continuing to grow [1],[2]. To allow measurements over sustained periods of time, body-worn sensors are required to have long lifetimes; hence, there is a need to power them effectively and efficiently. While mobile devices such as smartphones and media players have average power requirements of tens to hundreds of milliwatts, typical body sensors require only tens to hundreds of microwatts [3],[4]. Primary or secondary batteries are commonly used to satisfy these power demands. Batteries are energy constrained, that is, they are able to provide a finite amount of energy before they can no longer operate. Replacing or recharging batteries is often cumbersome and, in some cases, may not even be possible [4]. This results in devices having lifetimes limited by the battery capacity, which is typically relatively short due to a

Manuscript received March 06, 2014. This work was supported in part by the Engineering and Physical Sciences Research Council (EPSRC) UK under grant EP/G067740/1 "Next Generation Energy-Harvesting Electronics: Holistic Approach," www.holistic.ecs.soton.ac.uk

The authors are with Electronics and Computer Science, University of Southampton, SO17 1BJ, UK (phone: +44 (0)23 8059 2775; fax: +44 (0)23 8059 2901; e-mail: gvm@ecs.soton.ac.uk).

Copyright (c) 2013 IEEE. Personal use of this material is permitted. However, permission to use this material for any other purposes must be obtained from the IEEE by sending a request to pubs-permissions@ieee.org. necessity for unobtrusiveness and wearability (hence lightweight and miniature devices).

Recently, the potential for human-powered energy harvesting - extracting energy from the human body to power electronic devices - has been gaining increased attention [4]. These energy sources are power constrained, that is they can theoretically provide unlimited energy over an infinite period of time, yet only very limited power can be obtained at any particular time. Such human-powered energy harvesters (referred to as microgenerators) can extract energy from a range of sources [5], including body-heat [6], forces directly applied by the body (for example footfall [7], or joint movements [8][9]), or the inertia of the human body [10]. It is the latter of these that we focus on in this paper.

Human-powered inertial microgenerators have been proposed in the literature [11] and, while some are designed to operate off-resonance [12], the majority are resonant systems. As the frequencies present in movement of the human body are typically below $4 \mathrm{~Hz}$, a major challenge is posed to the design of resonant devices as their dimensions typically increase as the resonant frequency decreases [10]. Furthermore, the amplitudes and frequencies of these motions are not constant, requiring a microgenerator with a wide bandwidth to operate effectively. The majority of proposed microgenerators are sensitive to only forces in a single direction, referred to hereon as one degree-of-freedom (1DOF) microgenerators. More recently, designs have been proposed for generators that are able to harvest energy from more than one axis. Techniques using two degree-of-freedom (2-DOF) microgenerators (those that do not have a single axis of excitation, but instead can harvest energy from motion in a plane) have demonstrated increased energy generation [13],[14],[15].

A number of research studies have investigated the energy harvestable from the human body. While originally limited to the analysis of the energy that the body theoretical expends [16],[17], more recent studies have investigated the energy available from inertial forces during specific exercises and at a variety of locations on the body [18]. While many of these studies have considered contrived activities (for example a single 60-second period on a running machine) [19], others have considered datasets obtained over many days and hence representing common activities of daily living [3]. Many studies have considered performance variation between different participants [20], while others have considered the acceptable volume and weight at different mounting locations 

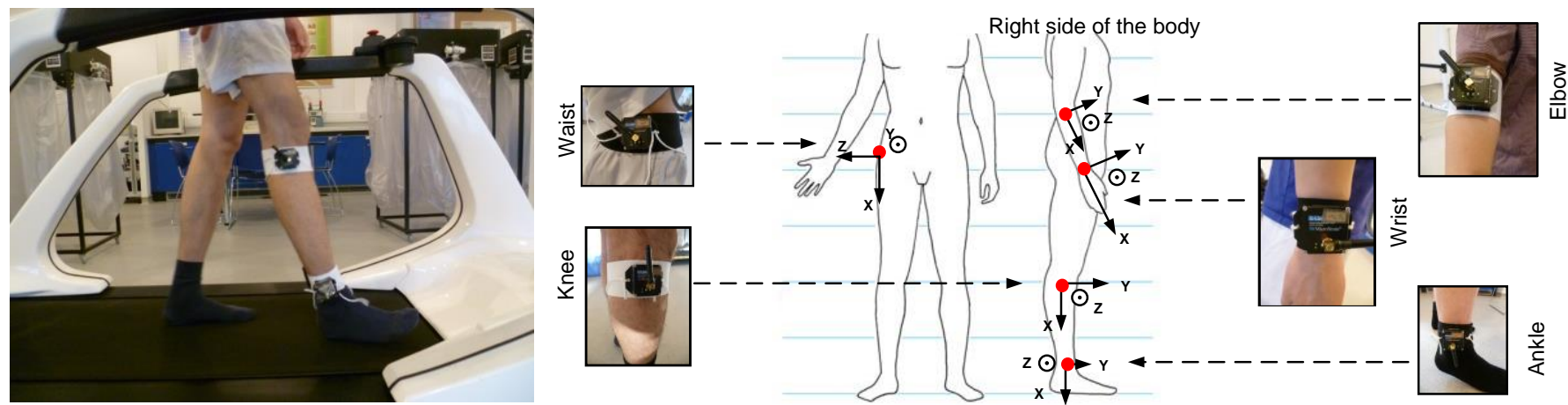

Fig. 1. Acceleration data being collected on a participant's right knee during walking on a treadmill (left), and the five data collection locations around the body showing the directions of the accelerometers' axes (right).

on the body [3]. While a rich analysis of the problem area has been performed, existing studies have only analyzed the available power in a limited number of sensitive directions; the most complete study independently considered each of the accelerometer's three Cartesian axes [3]).

In this paper, we consider the effect on harvestable power of generator location (five locations on the body), human activity (walking and running) and variation within a population (ten different participants). While previous studies have also considered these effects, this is the first paper to also consider the effect of generator orientation and its relation with the other parameters. Through experimentally obtained data, we show that orientation is a significant issue particularly if, as would usually be the case, generator placement is influenced by the wearer (and hence may be susceptible to user error and differences in body-shape). Furthermore, we propose and analyze how 2-DOF generators can alleviate these problems and improve a generator's tolerance to orientation variation and different activities. We also investigate their ability to provide consistent operation over a range of different wearers.

The structure of this paper is as follows. In Section II we explain the experimental methodology for obtaining acceleration data. Section III outlines the analysis process for evaluating the effect of orientation on harvestable power. Section IV presents results from this analysis, showing the effect of orientation, location and activity on harvestable power and comparing the effectiveness of 1-DOF and 2-DOF microgenerators. Section V draws conclusions and identifies areas of future work.

\section{EXPERIMENTAL DATA COLLECTION}

As previously outlined, the aim of this research is to investigate the effect of activity, location and orientation on the obtainable power from a human-powered inertial microgenerator. Furthermore, we wish to analyze these for both 1-DOF and 2-DOF microgenerators and compare the results to understand if a 2-DOF microgenerator allows greater tolerance to rotation (commonly caused as a result of orientation offset as a result of different body-shapes or misalignment by the user).

To undertake this investigation, an experiment was devised to record acceleration data from a number of participants while they performed walking and running exercises. Tri-axial acceleration data were collected from different locations on the body, while participants performed these activities. Once collected, these data were first processed to simulate the effect of rotating a microgenerator, and then analyzed to obtain the theoretical maximum power. Ethical approval for the study was obtained from the University's Electronics and Computer Science ethics committee under application N/10/10/002.

Ten participants ( 8 male, 2 female; aged 24-33yrs) were asked to run and walk on a treadmill. The limited age range sampled may affect the applicability of the results to a general population, however this would require further investigation to establish. In an attempt to ensure natural behavior, participants were asked to set the treadmill at a speed they found comfortable. This speed, and their resulting step rate (averaged over the full 30 second period), were recorded; the collected information is provided in Table 1.

While each participant performed the exercises, they wore wireless tri-axial accelerometers (Microstrain G-Link) sampling at $128 \mathrm{~Hz}$. Five key locations for mounting the accelerometers were identified from the literature [10],[20],[18], namely the ankle, knee, waist, elbow and wrist; these locations are illustrated in Fig. 1. The exact positions were defined with reference to physiological markers (for example bone and joint positions). Accelerometers were mounted using a Velcro strap (similar to a sweatband), as illustrated in Fig. 1. While this allowed some movement of the device, this is not dissimilar to variation encountered by any

TABLE 1

PARTICIPANT DETAILS RECORDED DURING DATA COLLECTION

\begin{tabular}{c|c|c|c|c|c|c}
\hline \hline \multirow{2}{*}{ Gender } & Age & Height & \multicolumn{2}{|c|}{$\begin{array}{c}\text { Step Freq (Hz) } \\
\text { (yrs) }\end{array}$} & \multicolumn{2}{c}{ Speed (kph) } \\
(cms) & Walk & Run & Walk & Run \\
\hline M & 28 & 179 & 1.9 & 2.5 & 4.6 & 8.6 \\
M & 24 & 176 & 1.7 & 2.5 & 3.5 & 6.0 \\
M & 28 & 182 & 1.7 & 2.6 & 3.2 & 7.4 \\
M & 33 & 185 & 1.7 & 2.7 & 3.0 & 6.4 \\
M & 24 & 175 & 1.7 & 2.6 & 3.8 & 8.0 \\
M & 24 & 190 & 1.7 & 2.5 & 2.7 & 4.5 \\
F & 23 & 160 & 2.0 & 2.9 & 3.0 & 5.5 \\
F & 24 & 168 & 1.8 & 2.7 & 4.5 & 7.0 \\
M & 25 & 178 & 1.7 & 2.5 & 3.0 & 7.5 \\
M & 24 & 190 & 2.0 & 3.1 & 5.0 & 7.5 \\
\hline Mean & 25.7 & 178 & 1.8 & 2.7 & 3.6 & 6.8 \\
STD & 3.1 & 9.4 & 0.13 & 0.19 & 0.81 & 1.2 \\
\hline \hline
\end{tabular}


wearable device mounted on the body which would likely be attached in a similar fashion. The accelerometers were attached by the researcher conducting the experiments.

To provide additional validation of our results, further acceleration data measured on the wrist, ankle and hip were obtained via The EH Network Data Repository (http://ehnetwork.org/data) [21], and included in this analysis. These data were collected using a different protocol, for example the accelerometer had a greater volume and weight, and the treadmill was operated only at predefined speeds.

\section{Calculating Obtainable POWER}

Once obtained using the method presented in Section II, triaxial acceleration data from different locations and activities were processed to estimate and analyze the power obtainable through inertial energy harvesting. This research does not consider specific energy harvesting devices. Therefore, to perform this analysis, the following assumptions are made:

- A linear resonant microgenerator is considered, allowing the fundamental equations for power [22] to be applied;

- Individual frequency components of acceleration data are considered sinusoidal (and hence SHM can be assumed);

- For simplicity, only frequency components at resonance are considered (equivalent to a high-Q microgenerator);

- A 1-DOF microgenerator is considered to harvest energy from only motion along a single axis.

- A 2-DOF microgenerator is considered to be formed from two orthogonally-mounted 1-DOF generators.

First, the power harvestable from a 1-DOF generator mounted in different orientations is estimated, thus also inferring the tolerance of existing microgenerators to variation in orientation. This analysis, described in Section III.A, allows easy interpretation of the data in different orientations. As explained in Section III.B, this method is not suitable for comparing the relative performance of both a 1-DOF and 2DOF microgenerator, hence an alternative method (for which results are not as intuitively interpretable) is proposed.

\section{A. Analyzing the Effect of Orientation}

Initially, the acceleration vector $\mathbf{a}(t)\left[\mathrm{ms}^{-2}\right]$ is formed from the output of the triaxial accelerometer as shown in (1), where

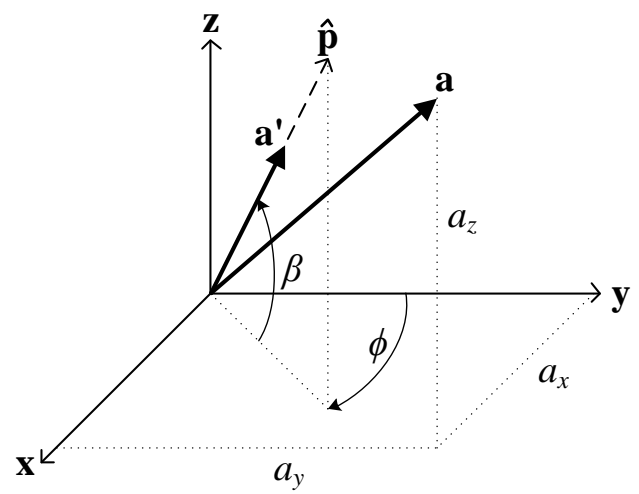

Fig. 2. The projection of the acceleration vector a onto the direction of unit vector $\widehat{\mathbf{p}}$. The unit vector $\widehat{\mathbf{p}}$ is defined by angles $\phi$ and $\beta$. $\hat{\mathbf{x}}, \hat{\mathbf{y}}$ and $\hat{\mathbf{z}}$ are unit vectors aligned with the accelerometer axes.

$$
\mathbf{a}(t)=\left(a_{x}(t) \hat{\mathbf{x}}, a_{y}(t) \hat{\mathbf{y}}, a_{z}(t) \hat{\mathbf{z}}\right)
$$

To evaluate different microgenerator orientations, a unit vector $\widehat{\mathbf{p}}$ is created (2):

$$
\widehat{\mathbf{p}}(\beta, \phi)=(\cos \beta \sin \phi \hat{\mathbf{x}}, \cos \beta \cos \phi \hat{\mathbf{y}}, \sin \beta \hat{\mathbf{z}})
$$

This vector represents the direction along which energy is harvested by a 1-DOF microgenerator (its 'sensitive' direction), as illustrated by Fig. 2 . The vector $\widehat{\mathbf{p}}$ is defined by angles $\beta$ and $\phi$ [degrees], defined by (3) and (4):

$$
\begin{gathered}
\beta \in 5 \cdot \mathbb{N}_{0}, \quad \beta \leq 90 \\
\phi \in 5 \cdot \mathbb{N}_{0}, \quad \phi \leq 360
\end{gathered}
$$

A $5^{\circ}$ increment is chosen to balance angular resolution with the time required to perform the complete analysis. By taking the scalar product of (1) and (2), the acceleration vector $\mathbf{a}$ is projected onto the direction of $\widehat{\mathbf{p}}$, as shown in (5).

$$
a^{\prime}(t, \beta, \phi)=\mathbf{a}(t) \cdot \widehat{\mathbf{p}}(\beta, \phi)
$$

This provides a measure of the magnitude of the acceleration in the sensitive axis of the microgenerator, where $a^{\prime}=\left\|\mathbf{a}^{\prime}\right\|$.

Next, $a^{\prime}$ is transformed into the frequency domain using an FFT, following which the frequency axis is split into discrete 'bands'. Each band $b$ (where $b \in 0.5 \mathbb{N}, 0<b<50$ ) crops the FFT so that it contains only frequencies in the range defined by $[b, b+0.5)$. For clarity, in this paper all frequency components in the range $[3,50) \mathrm{Hz}$ are grouped into a single band. For each band, the maximum amplitude $A_{b}\left[\mathrm{~ms}^{-2}\right]$ and its corresponding frequency $f_{b}[\mathrm{~Hz}]$ is extracted, and these values are used to calculate the obtainable power.

$$
P=\frac{A_{b}^{2} m}{8 \pi f_{b} \xi_{T}}
$$

Finally, the theoretical power obtainable in each frequency band can be calculated using (6), where $m$ is the generator mass $[\mathrm{kg}]$ and $\xi_{T}$ is the total damping ratio. In this paper, the term relative power $[\mathrm{W} / \mathrm{kg}]$ is used for ease of comparison, and is defined with $m / \xi_{T}$ set to unity (7).

$$
P_{r}=\frac{P \xi_{T}}{m}=\frac{A_{b}^{2}}{8 \pi f_{b}}
$$

For any one dataset obtained (for example, 30 seconds of acceleration data from a single participant's ankle while running) a direction, $\max (\widehat{\boldsymbol{p}})$, will be identifiable in which maximum relative power, $\max \left(P_{r}\right)$, is generated. Both of these values will vary between participants; for example an individual who has more pronounced movements would 


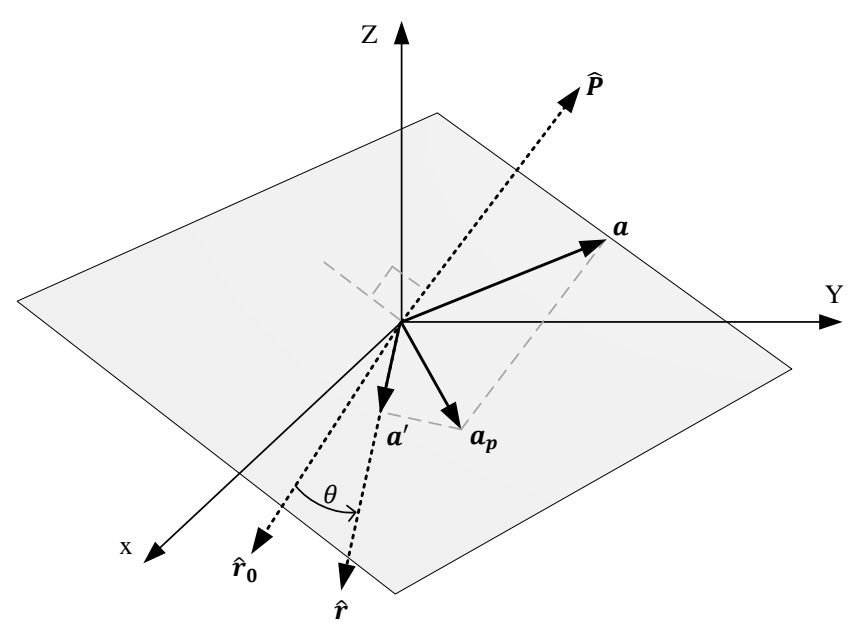

Fig. 3. The projection $\mathbf{a}_{p}$ of the acceleration vector $\mathbf{a}$ onto a plane ( $\perp$ to $\left.\widehat{\mathbf{p}}\right)$, and the acceleration magnitude of $a^{\prime}$ resulting from the subsequent rotation of $\mathbf{a}_{p}$ on this plane by an angle $\theta$.

expect to have comparatively larger acceleration magnitudes. In order to permit effective comparison of orientation between participants, a concept of normalized power is introduced (8).

$$
P_{n}=\frac{P_{r}(b)}{\max \left(P_{r}(b)\right)}
$$

Hence, for each dataset, the normalized power shows how much power an individual is generating with respect to the maximum that they are capable of.

\section{B. The Effect of Rotation in 2-DOF Microgenerators}

The analysis method shown Section A allows for easy interpretation of the effect of orientation on a 1-DOF microgenerator, as the influence of $\beta$ and $\phi$ intuitively map to the microgenerator's sensitive direction $\widehat{\mathbf{p}}$. However, to fully evaluate the effect of rotation on a 2-DOF microgenerator, consideration of an additional axis of oscillation is necessary.

As with the previous method, the output of the triaxial accelerometer is mapped to an acceleration vector $\mathbf{a}$, and angles $\beta$ and $\phi$ define a unit vector $\widehat{\mathbf{p}}$. However, the acceleration vector $\mathbf{a}$ is now projected onto the plane perpendicular to the unit vector $\widehat{\mathbf{p}}$ as $\mathbf{a}_{p}\left[\mathrm{~ms}^{-2}\right]$ (9), as illustrated by Fig. 3 .

$$
\mathbf{a}_{p}(t, \beta, \phi)=\widehat{\mathbf{p}}(\beta, \phi) \times(\mathbf{a}(t) \times \widehat{\mathbf{p}}(\beta, \phi))
$$

Next, a unit vector $\widehat{\mathbf{r}}_{0}$ (representing the sensitive direction of the harvester) is defined on this plane (10), (11), where $\hat{\mathbf{y}}$ is the unit vector along the $\mathrm{y}$-axis and $p_{z}$ is the component of the unit vector $\widehat{\mathbf{p}}$ in the z-axis.

$$
\begin{gathered}
\mathbf{r}_{0}(\beta, \phi)=\hat{\mathbf{y}} \times \widehat{\mathbf{p}}(\beta, \phi) \\
\hat{\mathbf{r}}_{0}(\beta, \phi)=\frac{\mathbf{r}_{0}(\beta, \phi)}{\left\|\mathbf{r}_{0}(\beta, \phi)\right\|}
\end{gathered}
$$

The unit vector $\hat{\mathbf{r}}_{0}$ is subsequently rotated about the normal vector $\widehat{\mathbf{p}}$ by an angle $\theta$ [degrees] such that:

$$
\hat{\mathbf{r}}(\beta, \phi, \theta)=\mathbf{R}_{\theta} \hat{\mathbf{r}}_{0}(\beta, \phi)
$$

where $\mathbf{R}_{\boldsymbol{\theta}}$ is a rotation matrix used to rotate counterclockwise about the vector $\hat{\mathbf{r}}_{0}$ by the angle of $\theta$ [degrees], defined by (13):

$$
\theta \in 10 \cdot \mathbb{N}_{0}, \theta<90
$$

A $10^{\circ}$ increment is chosen to balance angular resolution with the time taken for analysis. The projected acceleration vector $\mathbf{a}_{p}$ is subsequently projected onto $\hat{\mathbf{r}}$ using (14):

$$
a^{\prime}(t, \beta, \phi, \theta)=\mathbf{a}_{p}(t, \beta, \phi) \cdot \hat{\mathbf{r}}(\beta, \phi, \theta)
$$

This provides a measure of the magnitude of the acceleration in the sensitive axis of the microgenerator. From this, the data is transformed into the frequency domain and analyzed in discrete frequency bands as described by (6)-(8) in Section A.

In the case of a 2-DOF microgenerator, it is assumed that the two sensitive axes are perpendicular to each other. Hence, the obtainable power is calculated assuming that the second sensitive axis is offset by a rotation of 90 degrees on the plane, as shown by (15). The relative power obtainable from the 2DOF microgenerator, $P_{r}$, is the sum of powers available from each of the projected accelerations $a_{1}^{\prime}$ and $a_{2}^{\prime}$.

$$
\begin{gathered}
a_{1}^{\prime}(t, \beta, \phi, \theta)=a^{\prime}(t, \beta, \phi, \theta) \\
a_{2}^{\prime}(t, \beta, \phi, \theta)=a^{\prime}(t, \beta, \phi, \theta+\pi / 2)
\end{gathered}
$$

This section has presented the methods for evaluating acceleration data in order to analyze the effect of harvester orientation on obtainable power. The following section provides results and observations from analyzing collected acceleration data using these methods.

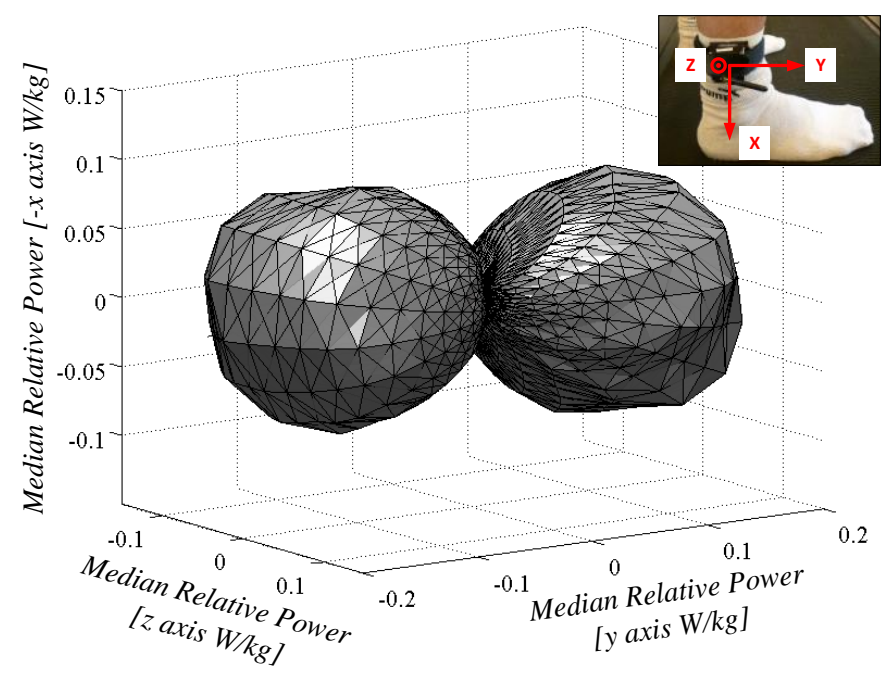

Fig. 4. Median relative power, $P_{r}$, obtainable from the ankle while walking, calculated across all participants and considering only frequency components within $0.5-1 \mathrm{~Hz}$. 


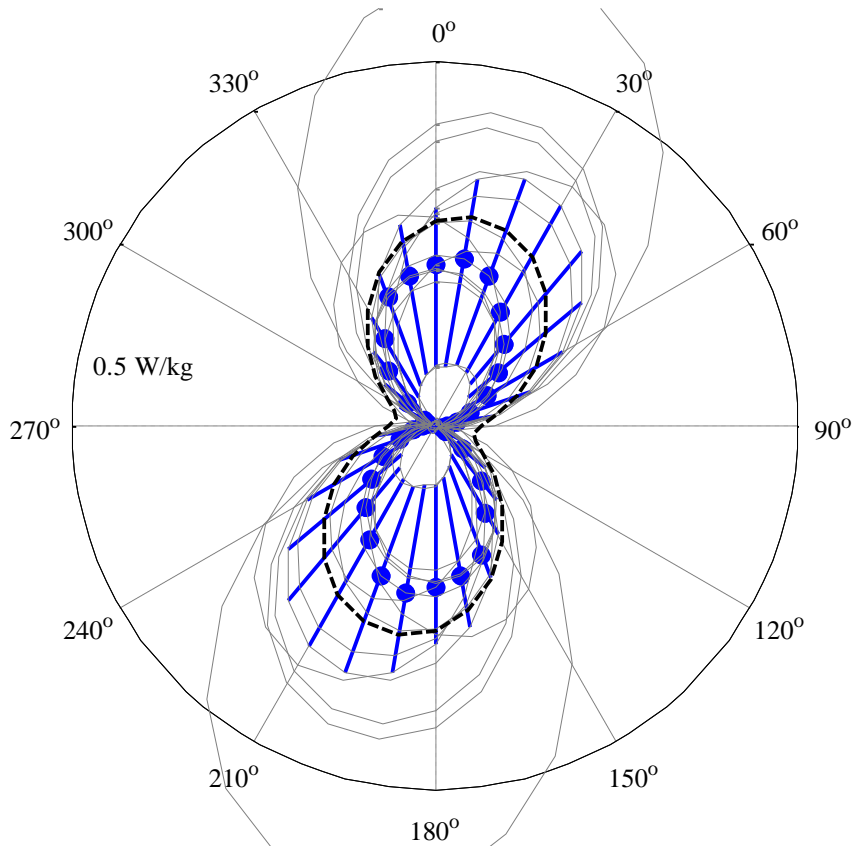

Fig. 5. Relative power obtainable (in the XY plane, rotating about angle $\phi$ ) from the ankle while walking, showing the median relative power $P_{r}$ (marked by filled circles) and interquartile range (IQR, denoted by the thick lines), and the individual data within the population (the thin, light grey lines). Relative power derived from the EH Network Data Repository [21] (the dashed line).

\section{RESULTS AND OBSERVATIONS}

This section discusses the results obtained through the experimental method and analysis techniques outlined in the previous sections. First, the results of the 1-DOF analysis are presented, followed by those of the 2-DOF analysis.

\section{A. 1-DOF Generator}

Using the collected data, the effect of 1-DOF harvester orientation was explored; the ankle location is used to illustrate this analysis process. Fig. 4 shows the median (across the entire sample population) relative power, $P_{r}$, that is obtainable with different harvester orientations. This illustrates that, as expected, the most energy can be harvested along the $y$-axis, i.e. the direction in which the foot moves.

Consider, as an illustration, that a cross-section is taken through the $z=0$ plane. The resulting cross-section is plotted in Fig. 5, where the median relative power $P_{r}$ is marked by filled circles, the interquartile range (IQR) is denoted by the thick lines, and the individual data for each member of the sample population are shown by thin, light grey lines. It is clear from the IQR that significant variation between participants is observed, hence limiting the ability to identify a statistically relevant 'optimal' orientation. However, while the magnitude of each $P_{r}$ trace varies considerably, the 'shape' of each remains reasonably consistent. The $P_{r}$ trace derived from the EH Network Data Repository [21] is shown by the dashed line, showing good correlation with our experimentally obtained data.

In Section III.A, we introduced the concept of normalized power. Comparing relative and normalized powers:

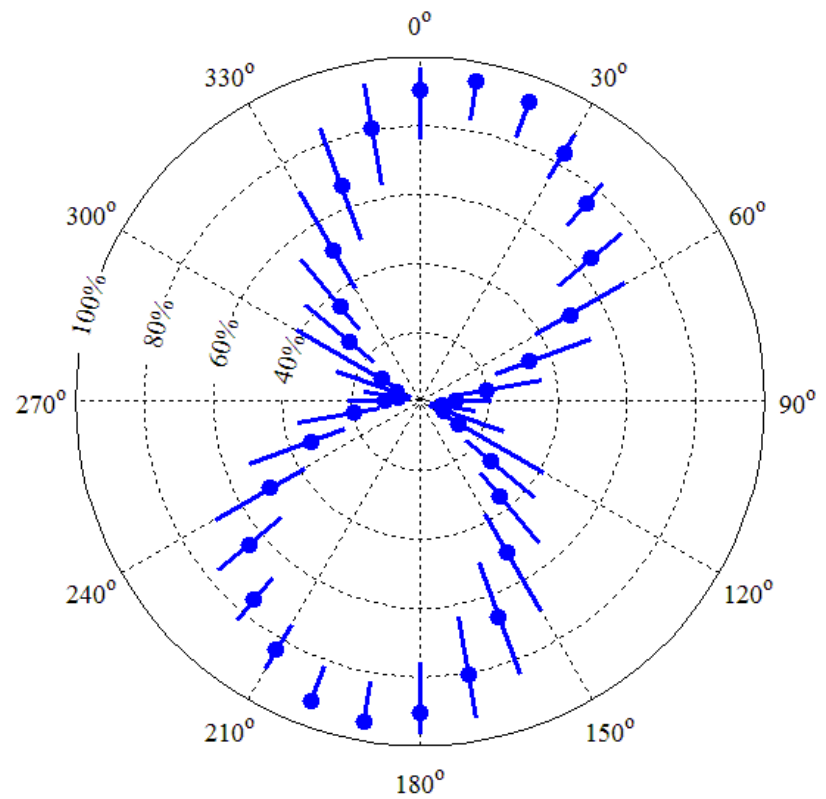

Fig. 6. Normalized power obtainable (in the XY plane, rotating about angle $\phi$ ) from the ankle while walking, showing the median normalized power $P_{n}$ (marked by filled circles) and interquartile range (IQR, denoted by thick lines).

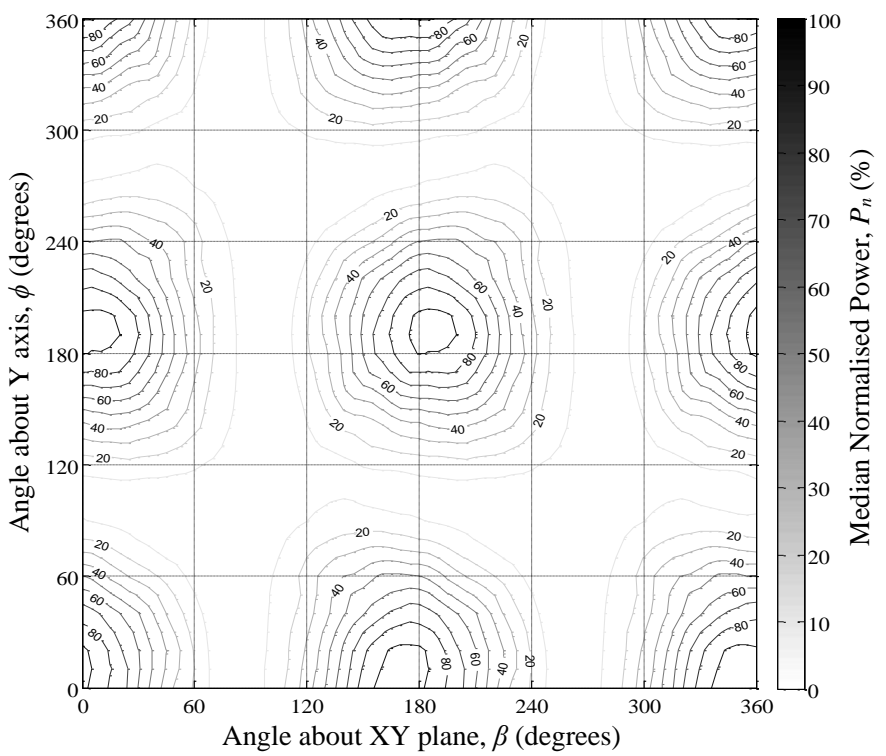

Fig. 7. Median normalized power $P_{n}$ obtainable from the ankle while walking with different generator orientations, calculated across all participants and considering only frequency components within $0.5-1 \mathrm{~Hz}$.

- Relative power is a measure of how much power an individual can harvest with a particular generator orientation, compared to everyone else in the population.

- Normalized power is a measure of how much power an individual can harvest with a particular generator orientation, compared to the best orientation for themselves.

Fig. 6 plots the same dataset as Fig 5 (i.e. acceleration in the $\mathrm{XY}$ plane, at the ankle during walking), but plotting the normalized power, $P_{n}$, rather than the relative power, $P_{r}$. In Fig. 6, filled circles represent the median normalized power across the population, while thick lines denote the IQR. This clearly shows that the IQR (and hence variation across the 


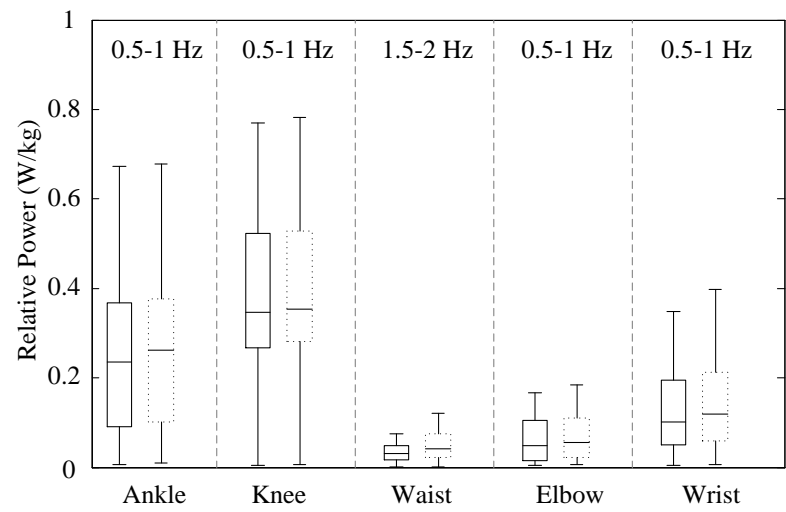

(a) Walking
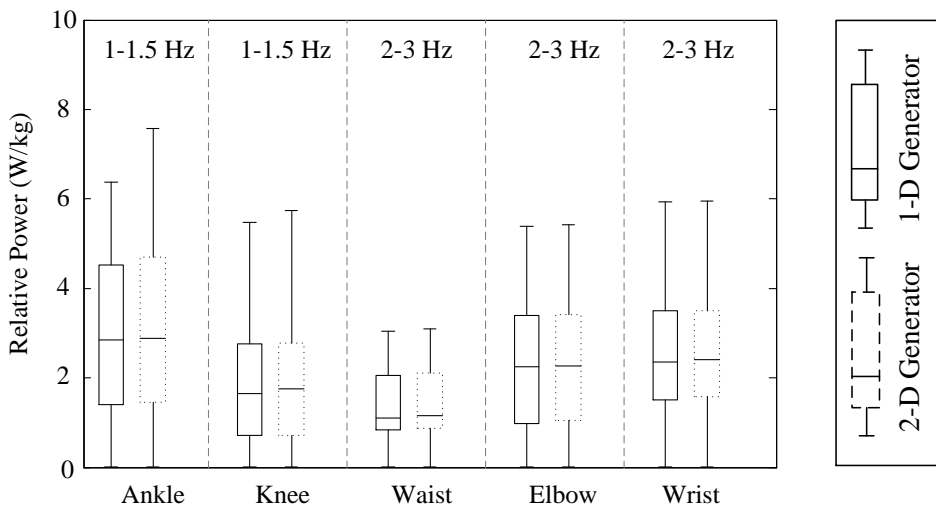

(b) Running

Fig. 8. Relative power available across different frequency ranges for (a) walking; and (b) running. The boxes show Q1 and Q3, while the central mark indicates the median, and the whiskers show lowest and highest datum still within 1.5. IQR of Q1 and Q3 respectively.

population) is considerably smaller for $P_{n}$ compared to $P_{r}$.

While Fig. 6 shows only the normalized power in the XY plane, Fig. 7 shows all possible orientations in 3-D space, through the vector defined by $\phi$ and $\beta$ (the data on Fig. 6 can be seen on Fig. 7 where $\beta=0$ ). The four repetitions of the peak can be expected, as the generator is sensitive to both 'backwards' and 'forwards' movements (hence duplicated by the regions $180 \leq \phi<360$ and $180 \leq \beta<360$ ). Through this analysis, it can be found that the preferred orientation (for our sample population), for a 1-DOF microgenerator mounted on the ankle, is $\phi=10^{\circ} \pm 20^{\circ}$ and $\beta=170^{\circ} \pm 20^{\circ}$. The tolerance on these indicates the acceptable deviation from this optimal orientation before the median $P_{n}$ drops by more than $10 \%$ (an arbitrary value chosen to indicate a noticeable drop in output power). This chosen orientation provides a combination of both high output power and angular sensitivity.

The preceding analysis has illustrated how the experimental process and analyses allow the investigation of optimal orientation for a 1-DOF energy harvester mounted on the ankle of a person walking, where only accelerations between $0.5-1 \mathrm{~Hz}$ were considered. However, as described in Section II, the experiments and analysis were also repeated for other exercises, locations on the body, and frequency ranges. Fig. 8 presents the median of the relative power $P_{r}$, obtainable in an optimal orientation, that can be harvested from different locations on the body. It also indicates the frequency band where the greatest output power was obtainable. Significant variation in relative power along the optimal orientations can be observed, which could be attributed to poor correlation between participants (as illustrated in Figs. 5 and 6).

When walking (Fig 8a), locations on the lower body (ankle and knee) generate a median relative power of 232-328 $\mathrm{mW} / \mathrm{kg}$, while location on the upper body (waist, elbow and wrist) generate $25-92 \mathrm{~mW} / \mathrm{kg}$. This illustrates that the harvestable output power from locations on the lower body is an order of magnitude higher than those on the upper body, due to the body's greater damping and reduced movement at locations closer to the head. When running (Fig. 8b), the relative power at the five locations is significantly increased, with a median relative power of $921-2590 \mathrm{~mW} / \mathrm{kg}$. The distinction between upper- and lower-body locations is less pronounced, as the upper body undergoes increased movement during vigorous activities.

For each location, Fig. 8 also shows the frequency range that the maximum relative power was available within. While running, the predominant frequency is $1.32 \mathrm{~Hz}$ on the lower body and $2.64 \mathrm{~Hz}$ on the upper body. This factor-of-two difference is because, on the upper body, the acceleration is a result of both feet striking the ground, and is hence at the step frequency. However, on the lower body, acceleration is a result of a single limb (where the generator is mounted on) impacting with the ground, and hence is at half the step frequency.

\section{B. 2-DOF Generator}

To investigate whether or not 2-DOF generators can improve the tolerance of a device to orientation and hence misalignment, a comparison between 1-DOF and 2-DOF inertial harvesters was made. Fig. 8 presents the median of the relative power from both 1-DOF and 2-DOF generators for comparison. The results show that use of a 2-DOF generator does not significantly increase the harvestable relative power, producing an average of only $18 \%$ greater power than the 1DOF equivalent. This is intuitive in the exercises considered

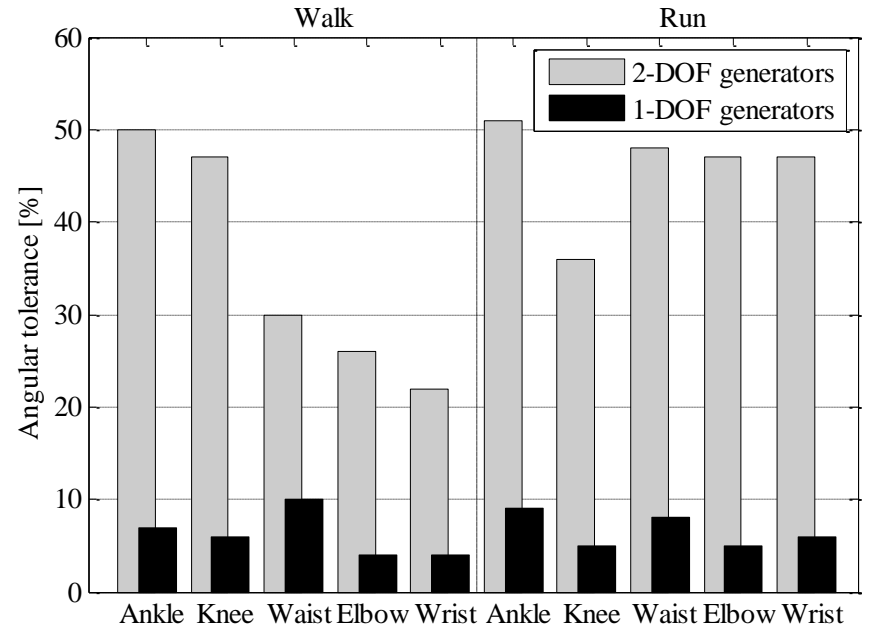

Fig. 9. Angular tolerance of 1-DOF and 2-DOF generators, at the five locations considered during both walking and running exercises. 
TABLE 2

NORMALIZED POWER OBTAINABLE FROM BOTH 1-DOF AND 2-DOF GENERATORS AT ALL LOCATIONS AND EXERCISES CONSIDERED.

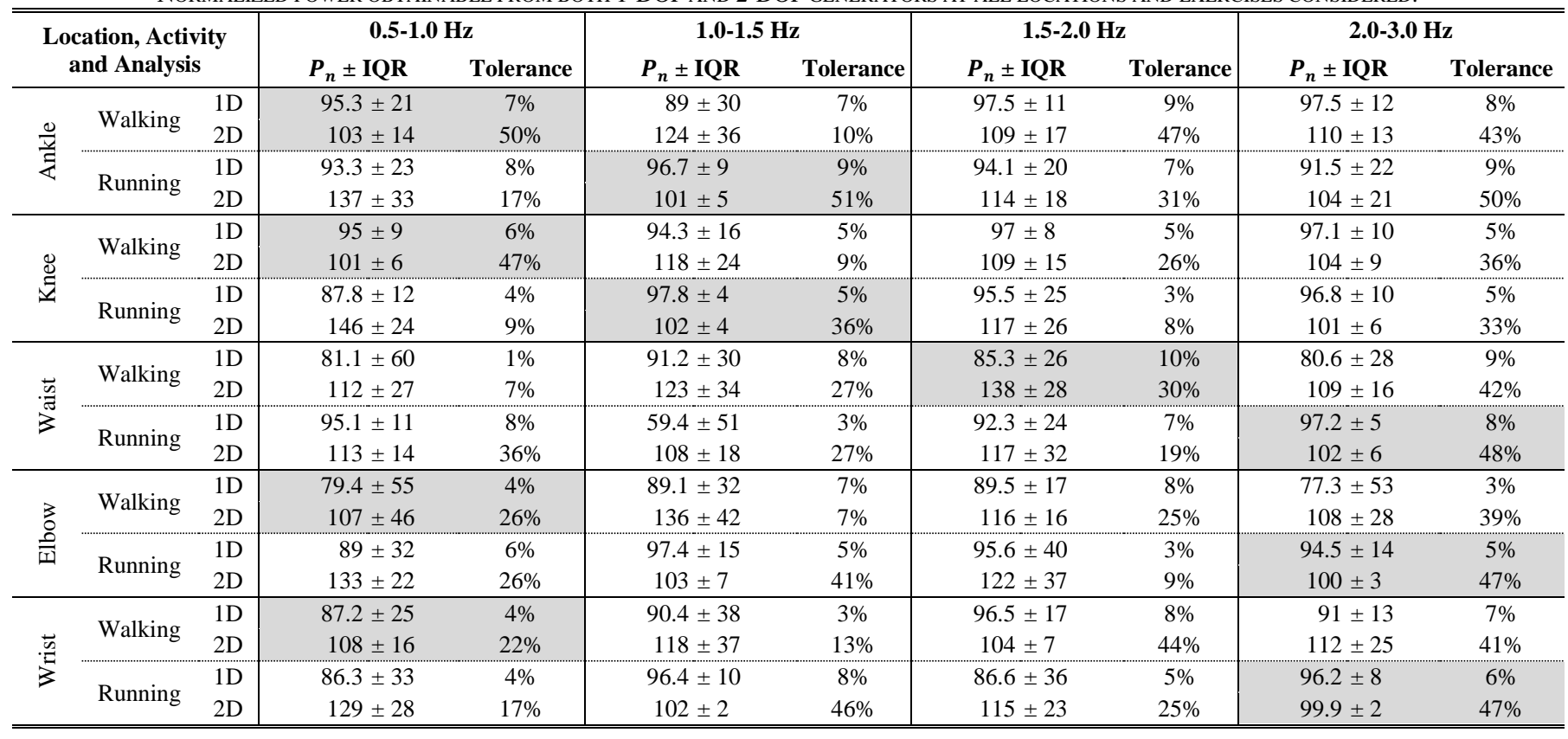

by this study, as it can be seen from Figs 6 and 7 that rotation by 90 degrees generally moves from a maximum to a minimum harvestable power.

However, the hypothesis of this work was not that a 2-DOF generator would provide greater power output, but that it would allow greater tolerance to rotation and misalignment. We use the term angular tolerance to refer to the sensitivity of a device's harvestable power to orientation, and define it as the percentage of orientations which result in a harvestable power that is $\geq 90 \%$ of the maximum relative power. Therefore, a larger angular tolerance reflects a potential to provide the rated power output despite deviating from the optimal orientation through misalignment and/or biomechanical variability. For comparison, the angular tolerance at different locations and exercises (considering only the predominant frequency band) for 1-DOF and 2-DOF generators is shown in Fig. 9. The results show that the angular tolerance of 2-DOF generators is $3-10 \times$ greater than that of 1-DOF generators. Across all locations and exercises, the 1-DOF generator provides an average tolerance of $6 \%$, whereas the 2-DOF case provides a tolerance of $40 \%$; this represents a $6 \times$ improvement.

For completeness, Table 2 presents the normalized power, $P_{n}$, obtainable from both 1-DOF and 2-DOF generators at all locations and exercises considered. For ease of comparison, the normalized power from a 2-DOF generator is normalized to the maximum relative power obtainable from their 1-DOF equivalent. Hence, a value exceeding $100 \%$ represents a 2 DOF generator which can harvest greater power than a 1-DOF generator. Results are shown for each of the frequency bands considered, and only the normalized power in the best orientation are included. The highlighted cells indicate the frequency band where the highest relative output power was generated, i.e. the results plotted in Fig. 9. In agreement with our previous conclusion, it can be clearly observed that a 2 DOF generator will provide a relatively small increase in output power compared to a 1-DOF generator, but will do so with significantly greater tolerance to variation in orientation.

\section{CONCLUSIONS AND FUTURE WORK}

This paper presented the results of an investigation into the effect of orientation, location and human activity on generated power. Our results correlate with those of both intuition and previous research, suggesting that 1) more vigorous activities/exercises result in greater harvested power (for example, running compared to walking), and 2) locations on the lower body provide more harvestable power (at least for exercises such as walking and running, where the lower-body experiences more energetic movement). It should be noted that, in the latter case, this effect is more pronounced during walking (where motion of the upper body is heavily damped) than running (where the upper body moves vigorously).

We have also shown, through substantial analysis, that harvestable power is significantly affected by rotation and misalignment, and hence proposed that 2-DOF generator designs have potential to reduce this effect. Our analysis and results indicate that 2-DOF generators do not provide significantly greater output power but, as suggested, can improve the tolerance to rotation by up to an order of magnitude.

We are currently validating the analytical results presented in this paper, by experimentally comparing the power output from both a 1-DOF and 2-DOF wearable energy harvester. To enable these experiments, we have designed and fabricated a low-frequency 1-DOF generator capable of harvesting energy from the low-frequency movement of the human body [23], and two of these can be mounted orthogonally to form a 2DOF generator. We are planning to evaluate the power output 
of both configurations at the different locations on the body, orientations and exercises presented in this paper, and will report on our findings in the future.

\section{ACKNOWLEDGMENT}

The authors would like to thank Dr Cheryl Metcalf and Martin Warner (Faculty of Health Sciences, University of Southampton) for their assistance in the participant study.

\section{REFERENCES}

[1] L. R. Pina, E. Ramirez, W. G. Griswold, "Fitbit+: a behavior-based intervention system to reduce sedentary behavior," in Int. Conf. Pervasive Computing Technologies for Healthcare (PervasiveHealth), 2012, pp.175-178.

[2] C. Edwards, C, "Healthcare's hi-tech lifelines," IET Engineering and Technology, vol.3, no.14, pp.36-39, Aug-Sep 2008.

[3] J. Yun; S. N. Patel, M. S. Reynolds, G. D. Abowd, "Design and performance of an optimal inertial power harvester for human-powered devices," IEEE Trans. Mobile Comput., vol.10, no.5, pp.669-683, May 2011.

[4] P. D. Mitcheson, E. M. Yeatman, G. K. Rao, A. S. Holmes, T. C. Green, "Energy harvesting from human and machine motion for wireless electronic devices," Proc IEEE, vol.96, no.9, pp.1457-1486, Sept. 2008.

[5] P. D. Mitcheson, "Energy harvesting for human wearable and implantable bio-sensors," in 32nd Annu. Int. Conf. IEEE Engineering in Medicine and Biology Society, Buenos Aires, Argentina, 2010.

[6] V. Leonov, "Thermoelectric energy harvesting of human body heat for wearable sensors," IEEE Sensors Journal, vol.13, no.6, pp.2284-2291, June 2013

[7] J. G. Rocha, L. M. Gonçalves, P. F. Rocha, M. P. Silva, S. LancerosMéndez, "Energy harvesting from piezoelectric materials fully integrated in footwear," IEEE Transactions on Industrial Electronics, vol. 57, no.3, pp. 813-819, March 2010.

[8] J. M. Donelan, Q. Li, V. Naing, J. A. Hoffer, D. J. Weber, A. D. Kuo, "Biomechanical energy harvesting: generating electricity during walking with minimal user effort," Science, vol.319, no.5864, pp.807-810, Feb. 2008.

[9] M. Pozzi, M. S. H. Aung, M. Zhu, R. K. Jones, J. Y. Goulermas, "The pizzicato knee-joint energy harvester: characterization with biomechanical data and the effect of backpack load," Smart Materials and Structures, vol.21, no.7, pp.075023, July 2012.

[10] T. von Büren, G. Tröster, "Design and optimization of a linear vibrationdriven electromagnetic micro-power generator," Sensors and Actuators A: Physical, vol.135, no.2, pp.765-775, Apr. 2007.

[11] C. R. Saha, T. O'Donnell, N. Wang, P. McCloskey, "Electromagnetic generator for harvesting energy from human motion," Sensors and Actuators A: Physical, vol.147, no.1, pp.248-253, Sept. 2008.

[12] M. Renaud, P. Fiorini, R. van Schaijk, C. van Hoof, "An impact based piezoelectric harvester adapted to low frequency environmental vibrations," in Int. Solid-State Sensors, Actuators and Microsystems Conf. (Transducers), 2009, pp.2094-2097.

[13] U. Bartsch, J. Gaspar, and O. Paul, "Low-frequency two-dimensional resonators for vibrational micro energy harvesting," Micromechanics and Microengineering, vol.20, no.3, pp. 1-12, 2010.

[14] Y. Zhu, S. O. R. Moheimani, M. R. Yuce, "A 2-DOF MEMS ultrasonic energy harvester," IEEE Sensors, vol.11, no.1, pp. 155-161, Jan. 2011.

[15] B. Andò, S. Baglio, F. Maiorca, C. Trigona, "Two dimensional bistable vibration energy harvester," in Eurosensors XXVI, Kraków, Poland, 2012, pp. 1061-1064.

[16] T. Starner, J. A. Paradiso, "Human-generated power for mobile electronics," in Low-Power Electronics Design, C. Piguet, Ed., FL: CRC Press, 2004, pp. 1-35.

[17] R. Riemer, A. Shapiro, "Biomechanical energy harvesting from human motion: theory, state of the art, design guidelines, and future directions," Journal of NeuroEngineering and Rehabilitation, vol. 8, no.22, 2011.

[18] A. Olivares, G. Olivares, P. Gloesekoetter, J. M. Górriz, J. Ramírez, "A study of vibration-based energy harvesting in activities of daily living," in Int. Conf. Pervasive Computing Technologies for Healthcare (PervasiveHealth), Munich, Germany, 2010.
[19] T. von Büren, P. D. Mitcheson, T. C. Green, E. M. Yeatman, A. S. Holmes, G. Troster, "Optimization of inertial micropower generators for human walking motion," IEEE Sensors, vol.6, no.1, pp.28-38, 2006.

[20] N. Yarkony, K. Sayrafian-Pour, A. Possolo, "Statistical modeling of harvestable kinetic energy for wearable medical sensors," in IEEE Int. Symp. World of Wireless Mobile and Multimedia Networks (WoWMoM), Montreal, QC, June 2010, pp.1-5.

[21] G. Szarka, A. S. Weddell, P. Proynov, B. Stark. (2011, October 24). University of Bristol/Southampton: Acceleration Data from Human Motion [Online]. Available: http://holistic.ecs.soton.ac.uk/resources.php

[22] S. P. Beeby, M. J. Tudor, N. M. White, "Energy harvesting vibration sources for microsystems applications," Measurement Science and Technology, vol.17, no.12, pp.R175-R195, 2006.

[23] H. Huang, G. V. Merrett, N. M. White, "Design of a linearized magnetic spring for body-worn inertial energy harvesters," in International Workshop on Algorithms and Concepts for Networked Sensing Systems Powered by Energy Harvesters (EnHaNSS'12), Antwerp, Belgium, June 2012.

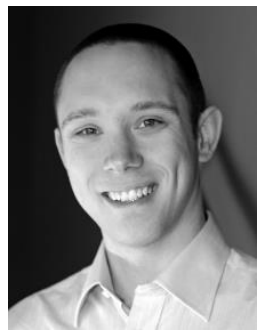

Geoff V. Merrett (GSM'06-M'09) received the B.Eng. degree (first class honors) in electronic engineering and the Ph.D. degree from the University of Southampton, UK, in 2004 and 2009 respectively. His thesis was titled 'Energy- and Information-Managed Wireless Sensor Networks: Modelling and Simulation.'

$\mathrm{He}$ was appointed as a Lecturer in energyefficient electronic systems at the University of Southampton in 2008, and was promoted to Associate Professor in 2014. He has published over 60 papers in journals and refereed conference proceedings, and contributed a chapter on 'Wireless Devices and Sensor Networks' to the book 'Energy Harvesting for Autonomous Systems' (Norwood, MA: Artech House, 2010). $\mathrm{He}$ has research interests in energy-efficient embedded systems, wireless sensing and sensor networks, and energy harvesting.

Dr Merrett is a member of the IET and Fellow of the HEA, and in 2013 received the IEEE award for Outstanding Student Branch Counselor. He reviews for many IEEE conferences and journals, and was General Chair of the Energy Neutral Sensing Systems (ENSsys) workshop in 2013 and 2014.

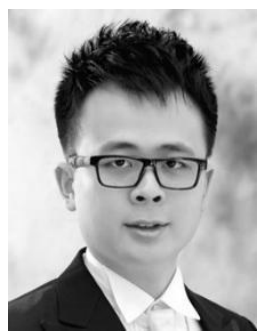

Hui Huang received a joint B.Eng. (Beijing University of Posts and Telecommunications, China) and B.Sc. (Queen Mary, University of London, UK) degree in 2009 in telecommunications engineering with management. He was awarded a Ph.D. from the University of Southampton, UK, in March 2014 for his thesis on 'Human-Powered Inertial Energy Harvesters: The Effect of Orientation, Location and Activity on the Obtainable Electrical Power'.

$\mathrm{He}$ is currently a Research Fellow in the Institute of Sound and Vibration Research at Southampton. His research interests are in energy harvesting, specifically from the motion of the human body, and electromechanical actuator development.

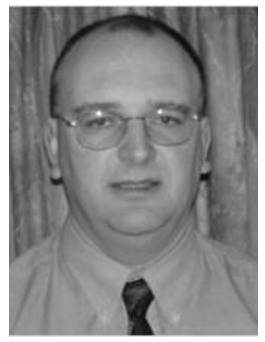

Neil M. White (M'01-SM'02) received a $\mathrm{PhD}$ degree from the University of Southampton, Southampton, UK, in 1988.

He holds a Personal Chair of Intelligent Sensor Systems in the School of Electronics and Computer Science, University of Southampton and is currently Head of School. He has published over 200 scientific papers in the area of sensors and instrumentation systems and holds 10 patents. $\mathrm{He}$ is a former Director and co-founder of the University spin-out company Perpetuum Ltd., which specializes in vibration energy harvesting.

Prof. White was the recipient of the 2009 Callendar silver Medal, awarded by the Institute of Measurement and Control for an 'outstanding contribution to the art of instruments and measurement'. He is a Chartered Engineer, Fellow of the IET, Fellow of the IOP and Chartered Physicist. 